\title{
Direct resolution of unoccupied states in solids via two-photon photoemission
}

\author{
W. Schattke, ${ }^{1,2}$ E. E. Krasovskii, ${ }^{1,3}$ R. Díez Muiño, ${ }^{2,4}$ and P. M. Echenique ${ }^{2,4,5}$ \\ ${ }^{1}$ Institut für Theoretische Physik und Astrophysik, Christian-Albrechts-Universität zu Kiel, Leibnizstraße 15, 24118 Kiel, Germany \\ ${ }^{2}$ Donostia International Physics Center DIPC, P. Manuel de Lardizabal 4, 20018 San Sebastián, Spain \\ ${ }^{3}$ Institute of Metal Physics, National Academy of Sciences of Ukraine, 03142 Kiev, Ukraine \\ ${ }^{4}$ Centro de Física de Materiales, Centro Mixto CSIC-UPV/EHU, Apartado 1072, 20080 San Sebastián, Spain \\ ${ }^{5}$ Departamento de Física de Materiales, Facultad de Químicas, UPV/EHU, Apartado 1072, 20080 San Sebastián, Spain
}

(Received 22 March 2008; revised manuscript received 11 August 2008; published 17 October 2008)

\begin{abstract}
Nonlinear effects in photoemission are shown to open an access to the band structure of unoccupied states in solids, totally different from hitherto used photoemission spectroscopy. Despite its second-order nature, strong resonant transitions occur, obeying exact selection rules of energy, crystal symmetry, and momentum. $A b$ initio calculations are performed to demonstrate that such structures are present in low-energy laser spectroscopy experimental measurements on Si. Similar resonances are predicted in ultraviolet angle-resolved photoemission spectra, as shown with a theoretical calculation on bulk Al.
\end{abstract}

DOI: 10.1103/PhysRevB.78.155314

PACS number(s): 79.60.-i, 71.15.-m, 71.20.-b

\section{INTRODUCTION}

A major challenge in theoretical condensed-matter physics is the description of excitations at the same level of accuracy as that reached in the description of the ground state. From the experimental point of view, information is limited; a direct momentum resolved band-structure spectroscopy for unoccupied states is available only through inverse photoemission spectroscopy (IPS). ${ }^{1,2}$ Despite a long history, IPS has never attained an accuracy comparable to that of angleresolved photoemission (ARPES) (Refs. 3 and 4) for occupied states. Other methods, such as electron diffraction at very low energies, ${ }^{5}$ are rather indirect. Furthermore, these methods are affected by the break of three-dimensional symmetry brought forth by the surface; a direct access to the band structure of the solid is thus prevented because of the cumbersome momentum nonconservation.

The new generation of broadly tunable light sources of high intensity developed in the last years can dramatically alter this situation. They have already fostered the nonlinear spectroscopies of atomic and molecular systems and opened the door to different and exciting subfields of physics, such as attosecond spectroscopy ${ }^{6}$ and quantum optics. ${ }^{7}$ In addition, multiphoton processes can be key to advance in the study of electronic excited states in solids. ${ }^{8}$ For crystals, both the intermediate and the final state of a multiphoton transition that obey the energy conservation rule are available over a wide photon energy range. This is in contrast to the resonance-enhanced multiphoton ionization technique (REMPI) widely used for atoms and molecules. The selectivity of the transition between bulk continuum states then solely depends on the wave functions. The multiphoton spectroscopy offers a unique possibility to perform band mapping, i.e., to determine the $\mathbf{k}$ space location of the waves involved in the surface perpendicular direction. This question does not arise for gas-phase molecular systems, and also in the solid-state multiphoton ARPES it did not receive much attention. Because little can be experimentally inferred about wave functions without support of a sound theory the twophoton measurements have concentrated on the energetically separated localized states in solids, such as surface and image states. ${ }^{9}$ In particular, the pump-probe techniques have been extensively employed for the study of the electron and adsorbate dynamics. ${ }^{10,11}$ At the same time, the multiphoton spectroscopy of the electronic states of the bulk continuum has received comparatively little attention. ${ }^{12-16}$ The role of intermediate unoccupied states in these transitions has not been fully understood yet. Theoretical models on this issue mainly rely on density-matrix time evolution ${ }^{17,18}$ and nonlinear-response treatments. ${ }^{19}$

In one-photon photoemission, the one-step model ${ }^{20}$ is known to provide a firm basis for $a b$ initio calculations. Experience shows that a high level of accuracy is absolutely necessary to interpret the experimental spectra even in this simpler case. ${ }^{21}$ One can expect the one-step model to reliably describe also a multiphoton case. Following the lines of the perturbation theory ${ }^{20}$ one arrives at the second-order contribution to the excited state arising from the initial state $|a\rangle$ of energy $\epsilon$

$$
|\epsilon+2 \hbar \omega\rangle=\hat{G}(\epsilon+2 \hbar \omega) \Delta \hat{H} \hat{G}(\epsilon+\hbar \omega) \Delta \hat{H}|a\rangle .
$$

Here $\Delta \hat{H}$ is the optical perturbation of frequency $\omega$, and $\hat{G}$ is Green's function of the system. The leftmost $\hat{G}$ operator is responsible for the transfer of the photoelectron to the detector. Initial and intermediate states are scattering states, which can be represented by Bloch waves (bulk wave functions) incident from the interior of the crystal and scattered by the surface. In addition, surface states, which are not related to any bulk solutions may appear among initial and intermediate states. Because we are interested only in the asymptotics of the final state at the detector, the summation over the final states with a given surface projection of the crystal momentum reduces to the calculation of the time-reversed lowenergy electron-diffraction (LEED) function, similar to the first-order one-step theory. The probability amplitude of detecting the electron at the energy $\epsilon+2 \hbar \omega$ is given by the expression $\langle\Phi|\Delta \hat{H} \hat{G}(\epsilon+\hbar \omega) \Delta \hat{H}| a\rangle$, in which the leftmost $\hat{G}$ operator has reduced to the time reversed LEED state $\langle\Phi|$. Owing to inelastic processes, the LEED function decays into 
the crystal, which is known to strongly broaden the peaks in the one-photon photoelectron spectra as a result of the relaxed momentum selection in the one-photon matrix element $\langle\Phi|\Delta \hat{H}| a\rangle$. In the two-photon case we have instead transitions from the initial to intermediate states, in which the Bloch constituents are not spatially damped: $\hat{G}(\epsilon$ $+\hbar \omega) \Delta \hat{H}|a\rangle$. [The inelastic effects appear in $\hat{G}(\epsilon+\hbar \omega)$ through complex energies.] The momentum selection rule then leads to much sharper peaks, which should be readily detected with tunable light sources of low bandwidth. This is the basic idea of a band-mapping procedure for unoccupied states in bulk, which circumvents the inaccuracies and drawbacks of the traditional approaches. In this paper we present its application at a level of accuracy similar to that usually reached in $a b$ initio calculations of one-photon photoemission spectra. The range of application of the scheme is far reaching, as it opens an access to the band structure of unoccupied states in solids. To prove our point, we analyze here two different sets of experimental photoemission spectra in $\mathrm{Si}^{12,13}$ and bring them together under one common interpretation.

\section{THEORETICAL MODEL}

Consider the spectral representation of the lowest-order terms. The probability per unit of time for transitions to a final state $|f\rangle$ (momentum $k$ and energy $E_{k}^{f}$ ) is given by a sum over occupied initial single-particle states $|a\rangle\left(k_{a}\right.$ and $\left.E_{k_{a}}^{a}\right)$ and unoccupied intermediate states $|z\rangle\left(k_{z}\right.$ and $\left.E_{k_{z}}^{z}\right)$

$$
\begin{aligned}
J \propto & \sum_{k_{a} \leq k_{F}}\left|h_{f a}\right|^{2} \delta\left(E_{k}^{f}-E_{k_{a}}^{a}-\hbar \omega\right)+\frac{1}{4} \sum_{k_{a} \leq k_{F}} \delta\left(E_{k}^{f}-E_{k_{a}}^{a}-2 \hbar \omega\right) \\
& \times\left|\sum_{k_{z}} \frac{h_{f z} h_{z a}}{E_{k_{z}}^{z}-E_{k_{a}}^{a}-\hbar \omega-i \eta}\right|^{2}
\end{aligned}
$$

Here real one-photon second-order emission processes are disregarded. The intermediate states $|z\rangle$ are reached by virtual one-photon transitions from the initial state observing Pauli exclusion principle. In contrast to the relaxed momentum conservation in the transition matrix elements $h_{f a}$, in the matrix elements $h_{z a}$ full three-dimensional momentum is conserved. ${ }^{22}$ It is this property which promises a large step forward in photoemission spectroscopy. First, because it allows the access to unoccupied states. Second, because it reaches the maximum resolution provided by first-principles theories.

The meaning of $\eta$ in Eq. (2) is twofold. First, it represents the decay width of the intermediate state as a lifetime and the dephasing of the transition amplitude owing to intrinsic elastic and inelastic processes. Second, the strong external photon field may broaden the energies by its ponderomotive force in high-intensity light sources. Even for small laser intensities, the ratio of two-photon to one-photon intensity is still significant for small broadening widths of some meV.

\section{TWO-PHOTON PHOTOEMISSION SPECTRA}

\section{A. Two-photon photoemission from $\mathrm{Si}(100)$}

As a first application of our formalism, we focus into photoemission processes triggered by strong optical laser fields in the femtosecond range. We address here two-photon photoemission processes from the technologically significant $\mathrm{Si}(001)$ semiconductor surface. Resonant photoexcitation in this surface has been already observed experimentally. ${ }^{12,13}$ In Ref. 12, a two-photon bulk transition was identified. The strong enhancement of the photoemission yield, with a peak width of $0.3 \mathrm{eV},{ }^{12}$ must be attributed entirely to an intermediate state. Furthermore, a strong peak from a two-photon process is observed in Ref. 13 as a transition from the $\Delta_{2}$, valence band to the $\Delta_{5}$ conduction band. This structure could be a candidate for the transitions discussed here as well. In any case, isolated single resonances are not required for our analysis. In the bulk continuum, the required energy matching between the photon energy and each one of the electron transitions occurs over finite-energy intervals, which contributes to the broadening of the peaks.

We first calculate the Si band structure (see Fig. 1) using density-functional theory (DFT) and the augmented planewave (APW) formalism. ${ }^{21}$ The two-photon photoemission spectra shown in Fig. 1 for two different values of photon energy (3.6 and $3.8 \mathrm{eV}$ ) are derived from this DFT calculation. The LEED state is calculated $a b$ initio assuming a steplike surface barrier and an optical potential $V_{i}$. Also the initial states are calculated as eigenfunctions of the semiinfinite crystal, but the wave functions are not spatially damped. In the present calculation we introduce certain approximations for intermediate states. In calculating the squared modulus of the matrix element $h_{z a}$, we sum up incoherently over all Bloch constituents, thus assuming an absolutely rough surface. The matrix elements $h_{f z}$ are also not calculated explicitly; they are assumed to depend only on the momentum difference $k_{f}-k_{z}$, through a Lorentzian function. This approximation affects relative intensities of different peaks in the energy distribution curve (EDC), and it is justified in so far as there is only one peak in the EDC (see Fig. 2). The width of the Lorentzian function is obtained from the imaginary part of the Bloch vector of the given LEED state in the presence of the imaginary potential $V_{i}$. The above approximations would be too rough in the less selective onephoton spectroscopy, but they are expected to be less crucial here in view of the sharp momentum conservation in the $|a\rangle \rightarrow|z\rangle$ transition.

Figure 1 shows that distinct peaks in the two-photon photoemission yield are associated with initial to intermediatestate resonances, corresponding to momentum-conserving transitions. In addition, the spectra show larger enhancements when the final wave function includes a large outgoing wave component, as denoted by the thickness of the green lines. This is further illustrated by the $a b$ initio calculations of the two-photon photoemission yield for the $\mathrm{Si}(001)$ surface, shown in Fig. 2. The theoretical yield of Fig. 2 very much resembles that measured in Ref. 12, assuming an overall shift of the theoretical unoccupied states by $0.3 \mathrm{eV}$ to higher energy, within the picture of a scissor operator. Ap- 


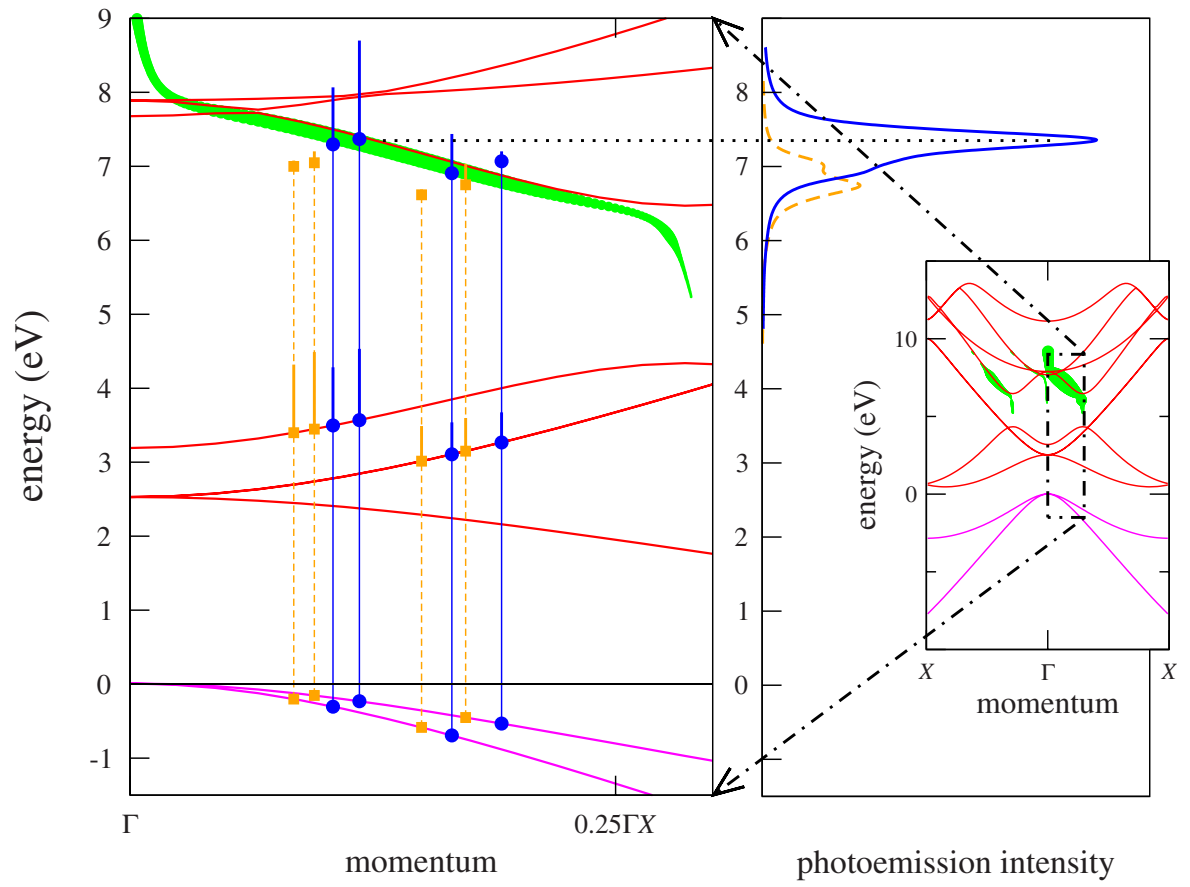

FIG. 1. (Color online) Two-photon photoemission yield and some relevant two-photon transitions in Si(001). Right panel: photoemission intensity vs energy (relative to valence-band maximum). Orange dashed line (blue/dark gray solid line) corresponds to the spectrum for $\hbar \omega=3.6(3.8) \mathrm{eV}$. The full band structure is shown in the inset as well. Thickened green lines denote the complex band-structure constituents of the LEED states; the thickness gives the contribution of the bulk Bloch wave to the outgoing photoelectron wave function. Left panel: magnified view of a portion of the band structure that contributes to the photoemission spectrum. The transitions are indicated by squares and circles for $\hbar \omega=3.6$ and $3.8 \mathrm{eV}$, respectively. LEED states denoted as in right panel. Vertical bars rising from the squares and circles show the magnitude of the coupling matrix element for this transition.

plying the scissor operator, one can see that the maximum of the photoemission yield peaks shifts by approximately 0.15 $\mathrm{eV}$, to photon energy of $3.95 \mathrm{eV}$, as in experiment. The binding energies given by the peaks themselves then align, too. This proves the possibility to precisely adjust the energies to experiment.
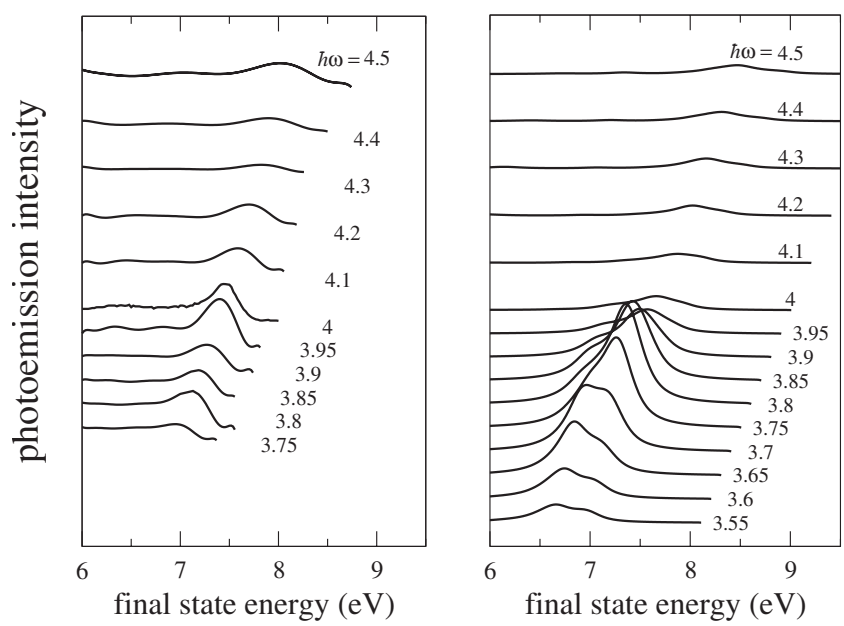

FIG. 2. Two-photon spectra from $\mathrm{Si}(001)$. Left panel: experimental values (Ref. 12) for $\hbar \omega=3.75-4.5 \mathrm{eV}$. Right panel: onestep calculation for $\hbar \omega=3.55-4.5 \mathrm{eV}$, with broadening $\eta$ $=0.15 \mathrm{eV}$ for initial and intermediate states, and optical potential $V_{i}=0.125 \mathrm{eV}$.
The calculated peak magnitude and dispersion are shown in Fig. 3 and compared to the corresponding measured magnitudes, ${ }^{12,13}$ finding good agreement as well. The energy

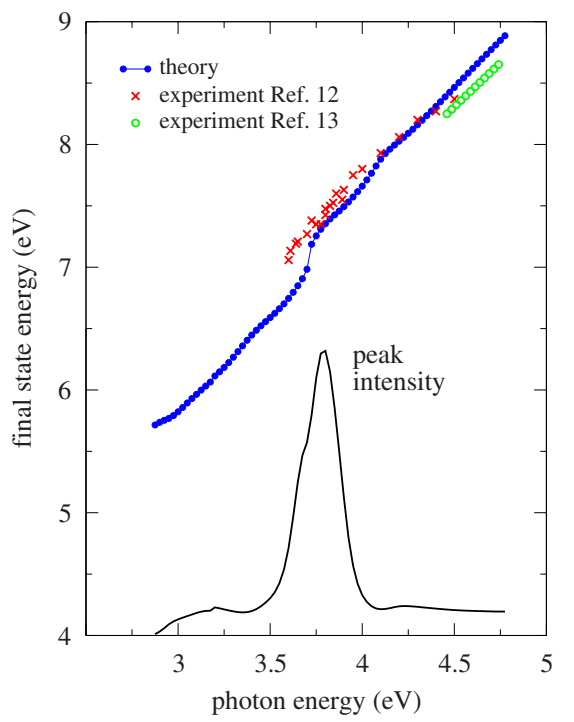

FIG. 3. (Color online) Peak magnitude and dispersion in the two-photon spectra of $\mathrm{Si}(001)$. Calculated (full blue/dark gray circles) peak positions are shown as a function of photon energy. Experimental values of Ref. 12 (red crosses) and Ref. 13 (open green/light gray circles) are shown as well. Solid black curve shows the theoretical peak height in arbitrary units. 


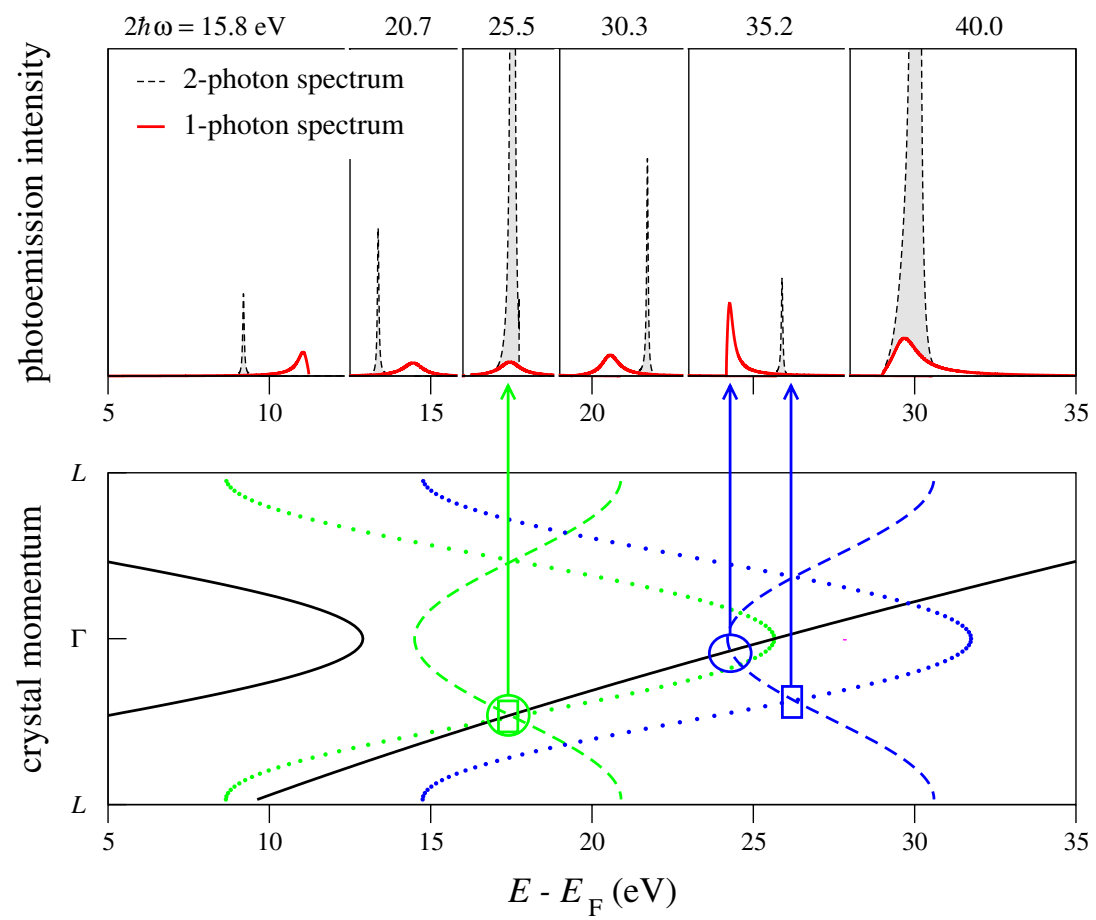

FIG. 4. (Color online) Upper panel: two-photon (broken gray lines) and one-photon (solid red/gray lines) photoemission yields for $\mathrm{Al}(111)$ vs binding energy. Six pairs of spectra corresponding to six different photon energies are shown. The numbers over the corresponding pairs of spectra denote twice the photon energy in units of eV. One-photon transitions are associated with doubled photon frequency. Lower panel: momentum vs energy of model band structure (solid lines). Two particular cases $[2 \hbar \omega=25.5$ (green/light gray) and $35.2 \mathrm{eV}$ (blue/dark gray)] are associated by arrows with the corresponding peaks in the spectra above. The initial band has been shifted by $2 \hbar \omega$ (broken lines) and the intermediate-state band has been shifted by $1 \hbar \omega$ (dotted lines). Rectangles (circles) denote conservation of momentum between initial and intermediate (initial and final) states.

analysis shows an almost linear final-state energy vs photon energy behavior. A kink is predicted at the photon energy for which the maximum peak intensity is found. This is precisely the energy at which the propagating final-state Bloch wave starts to contribute strongly to the LEED state. In other words, an electron in this state acquires a high probability to escape into vacuum.

\section{B. Two-photon photoemission from $\mathrm{Al}(111)$}

In addition to laser-based techniques, other standard photoemission techniques, such as ARPES, can benefit from the analysis proposed in this paper. For illustration purposes, we show in the following a model calculation on $\mathrm{Al}(111)$, in which photoemission spectra are obtained from a similar one-step calculation. We represent the $\mathrm{Al}(111)$ surface by a one-dimensional model in which the initial and intermediate states are tight-binding orbitals and the final states are nearly-free-electron waves. Only one branch of the final-state bands, which correspond to the LEED complex band structure that allows electron escape, is considered. We also use this system to discuss the expected magnitude of the nonlinear peaks, as compared to the one-photon peaks.

The upper panel of Fig. 4 groups six sets of two-photon photoemission spectra $(\eta=0.1 \mathrm{eV})$ together with six sets of one-photon photoemission spectra of doubled frequency. The final states for both types of photoemission processes are shown by the black solid line in Fig. 4. In each two-photon spectrum, a very narrow peak appears that dominates the spectrum. It comes from rather sharp transitions from the initial to the intermediate state (denoted by rectangles in the lower panel), given by the Lorentzian function in Eq. (2), which has the same shape as an exactly direct transition at a definite momentum. The intermediate to final-state transition is often obscured by the nonconservation of perpendicular momentum. If, by chance, it is conserved too, a resonant magnification of the peak occurs. In Fig. 4, this happens for $2 \hbar \omega=25.5 \mathrm{eV}$ and $2 \hbar \omega=40 \mathrm{eV}$.

Furthermore, in the example of the $2 \hbar \omega=35.2 \mathrm{eV}$ curve in Fig. 4, the rectangle shows the main peak resonance occurring at a momentum different from the usual one-photon direct transition into the final-state parabola. As a consequence, the photoemission yield is significantly reduced as compared to the transitions that fully conserve energy and momentum. A side peak arises at conserved momentum between the initial and final states (denoted by circles), as in one-photon spectra. The intermediate energy is not at its resonance, however. Without exceptions, the initial and final energies are sharp because of the $\delta$ function in Eq. (2).

\section{SUMMARY AND CONCLUSIONS}

Summarizing, we propose a methodology to extract momentum-resolved band-structure information for unoccupied electron states in bulk. The nonlinear contributions of 
the two-photon photoemission experimental spectra are used for this purpose. We show that the composition of a bulk two-photon spectrum displays the clear fingerprint of a Lorentzian line at exact momentum conservation for the transition between initial and intermediate state. It allows a direct association of both contributing bands and the determination of their energy difference at that specific momentum if the bands are unique. A standard Lorentzian deconvolution is necessary in the case of multiple bands. Experimental results for low photon energies on $\mathrm{Si}(001)$ support the theoretical findings. With respect to exploring and utilizing these effects in ARPES, the counterplay between higher light intensity (higher photoemission yield) and the concomitant broadening of the initial- to intermediate-state transition by ballistic acceleration has to be considered. Furthermore, a dispersion of the initial band sufficient to fix the momentum of that transition is needed.

Within this methodology, the location of fundamental band gaps in semiconductors can be directly seen in the two- photon spectra. Optical direct band gaps can be probed at any momentum where the transition via an intermediate state is observed. They are in contrast with the band gaps observed in direct and inverse photoemission, where the particle number in the solid is not maintained. The relaxation of the hole left behind in photoemission differs basically from that of the hole in an optical excitation. Thus the scissors operator, which rather artificially shifts quasiparticle energies can be extracted from the experiment. In $\mathrm{Si}(001)$, for instance, the real part of the self-energy could be adjusted to experiment as $0.3 \mathrm{eV}$. This shift seems to account for both a quasiparticle shift upward and an excitonic shift downward.

\section{ACKNOWLEDGMENTS}

We are grateful to Hrvoje Petek for a critical reading of the manuscript. Financial support by DFG under Contract No. FOR353 and the Spanish MEC (Grant No. FIS200766711-C02-02) is acknowledged.
${ }^{1}$ F. J. Himpsel and Th. Fauster, J. Vac. Sci. Technol. A 2, 815 (1984).

${ }^{2}$ R. Claessen, B. Burandt, H. Carstensen, and M. Skibowski, Phys. Rev. B 41, 8270 (1990).

${ }^{3}$ S. Hüfner, in Photoelectron Spectroscopy, Springer Series in Solid State Sciences Vol. 82 (Springer, Berlin, 1995).

${ }^{4}$ W. Schattke and M. A. Van Hove, Solid-State Photoemission and Related Methods (Wiley, New York, 2003).

${ }^{5}$ E. E. Krasovskii, W. Schattke, V. N. Strocov, and R. Claessen, Phys. Rev. B 66, 235403 (2002).

${ }^{6}$ R. Kienberger, E. Goulielmakis, M. Uiberacker, A. Baltuska, V. Yakovlev, F. Bammer, A. Scrinzi, Th. Westerwalbesloh, U. Kleineberg, U. Heinzmann, M. Drescher, and F. Krausz, Nature (London) 427, 817 (2004).

${ }^{7}$ C. S. Adams and E. Riis, Prog. Quantum Electron. 21, 1 (1997).

${ }^{8}$ H. Petek and S. Ogawa, Prog. Surf. Sci. 56, 239 (1997).

${ }^{9}$ Th. Fauster and W. Steinmann, in Photonic Probes of Surfaces, edited by P. Halevi (North-Holland, Amsterdam, 1995), Vol. 2, p. 347.

${ }^{10}$ U. Höfer, I. L. Shumay, Ch. Reuß, U. Thomann, W. Wallauer, and Th. Fauster, Science 277, 1480 (1997).

${ }^{11}$ H. Petek, M. J. Weida, H. Nagano, and S. Ogawa, Science 288, 1402 (2000).

${ }^{12}$ K. I. Shudo and T. Munakata, Phys. Rev. B 63, 125324 (2001).

${ }^{13}$ C. Kentsch, M. Kutschera, M. Weinelt, Th. Fauster, and M.
Rohlfing, Phys. Rev. B 65, 035323 (2001).

${ }^{14}$ G. Ferrini, C. Giannetti, G. Galimberti, S. Pagliara, D. Fausti, F. Banfi, and F. Parmigiani, Phys. Rev. Lett. 92, 256802 (2004); F. Banfi, C. Giannetti, G. Ferrini, G. Galimberti, S. Pagliara, D. Fausti, and F. Parmigiani, ibid. 94, 037601 (2005).

${ }^{15}$ N. Pontius, V. Sametoglu, and H. Petek, Phys. Rev. B 72, 115105 (2005).

${ }^{16}$ F. Bisio, M. Nývlt, J. Franta, H. Petek, and J. Kirschner, Phys. Rev. Lett. 96, 087601 (2006).

${ }^{17}$ T. Hertel, E. Knoesel, A. Hotzel, M. Wolf, and G. Ertl, J. Vac. Sci. Technol. A 15, 1503 (1997).

${ }^{18}$ H. Ueba and T. Mill, Appl. Phys. A: Mater. Sci. Process. 71, 537 (2000).

${ }^{19}$ C. Timm and K. H. Bennemann, J. Phys.: Condens. Matter 16, 661 (2004).

${ }^{20}$ P. J. Feibelman and D. E. Eastman, Phys. Rev. B 10, 4932 (1974).

${ }^{21}$ E. E. Krasovskii, K. Rossnagel, A. Fedorov, W. Schattke, and L. Kipp, Phys. Rev. Lett. 98, 217604 (2007).

${ }^{22}$ In one-photon photoemission, the uncertainty in perpendicular momentum $\Delta k_{\perp}$ can be estimated from the optical potential $V_{i}$ as $\Delta k_{\perp}=\left(2 V_{i}\right)^{1 / 2}$, whereas in two-photon photoemission the only limits to the full conservation of momentum are those linked to the optical skin depth. 